\title{
DECOMPOSITIONS OF $E^{3}$ WITH A COMPACT 0-DIMENSIONAL SET OF NONDEGENERATE ELEMENTS $\left({ }^{1}\right)$
}

\author{
BY \\ STEVE ARMENTROUT
}

1. Introduction. The purpose of this paper is to study monotone upper semicontinuous decompositions $G$ of $E^{3}$ such that the image, under the projection map, of the union of all the nondegenerate elements of $G$ is contained in a compact 0 -dimensional set. Such decompositions are of interest since a number of examples that have been studied [4], [6], [7], [9], [15] satisfy the conditions imposed above on $G$.

Suppose then that $G$ is a monotone upper semicontinuous decomposition of $E^{3}$. Let $E^{3} / G$ denote the associated decomposition space and let $P$ denote the projection map from $E^{3}$ onto $E^{3} / G$. Let $H_{G}$ denote the union of all the nondegenerate elements of $G$. Suppose that $P\left[H_{G}\right]$ is contained in a compact 0-dimensional set.

In $\S \S 3$ and 4 , point-like decompositions are considered. In $\S 4$, we prove that if $G$ is point-like and $E^{3} / G$ is a 3-manifold, then $E^{3} / G$ is homeomorphic to $E^{3}$. This settles a special case of a question raised by Bing in [7]. Other special cases of this question have been settled in [1], [2], [14], and [19]. Also in $\$ 4$, we give a condition which is both necessary and sufficient in order that $E^{3} / G$ be homeomorphic to $E^{3}$ in case $G$ is a point-like decomposition satisfying the conditions above. This condition is in terms of the existence of homeomorphisms from $E^{3}$ onto $E^{3}$ that shrink the nondegenerate elements of $G$ to small size.

In $\S \S 5$ and 6 , we study the following question: Is it true that if $G$ satisfies the conditions imposed above and $E^{3} / G$ is homeomorphic to $E^{3}$, then each element of $G$ is point-like? It is known [12] that if the set of nondegenerate elements is countable, the question has an affirmative answer. Furthermore, there is an example due to Bing [10, p. 7] of a monotone decomposition $B$ of $E^{3}$ into nonpoint-like sets such that $E^{3} / B$ is homeomorphic to $E^{3}$. In this case, $P\left[H_{B}\right]$ is an arc. Consequently, the condition imposed above on $P\left[H_{G}\right]$ cannot be omitted completely if an affirmative answer to the question is to be obtained. Although we do not settle this question, we give some partial affirmative solutions. We

Presented to the Society, August 28, 1964; under the title Point-like decomposition of $E^{3}$ with compact 0 -dimensional set of nondegenerate elements; received by the editors February 16, 1965.

(1) This research was partially supported by Air Force Office of Scientific Research Grant AF-AFOSR-359-63. 
give a solution in case each nondegenerate element of $G$ is an arc, or, in fact, any tree-like continuum. Additional partial solutions of this question are given in [3] and [13].

In $\S 7$, we give a number of special results concerning point-like decompositions $G$ of $E^{3}$ such that $E^{3} / G$ is homeomorphic to $E^{3}$.

2. Notation and terminology. If $X$ is a topological space and $G$ is an upper semi-continuous decomposition of $X$, then $X / G$ denotes the associated decomposition space, $P$ denotes the projection from $X$ onto $X / G$, and $H_{G}$ denotes the union of all the nondegenerate elements of $G$.

The statement that the upper semicontinuous decomposition $G$ of $E^{3}$ is monotone means that each set of $G$ is a compact continuum. A compact continuum $K$ in $E^{3}$ is point-like if and only if $E^{3}-K$ is homeomorphic to $E^{3}-\{0\}$. By a pointlike decomposition of $E^{3}$ is meant a monotone decomposition of $E^{3}$ into point-like sets.

If $n$ is a positive integer, the statement that $M$ is an $n$-manifold means that $M$ is a separable metric space, each point of which has a neighborhood $U$ in $M$ such that $U$ is an open $n$-cell. The statement that $M$ is an $n$-manifold-withboundary means that $M$ is a separable metric space such that each point of $M$ has a neighborhood $U$ in $M$ such that $U$ is an $n$-cell. If $M$ is an $n$-manifoldwith-boundary, then the boundary of $M$, denoted by $\mathrm{Bd} M$, is the set of all points $p$ of $M$ wich do not have open $n$-cell neighborhoods in $M$, and the interior of $M$, Int $M$, is $M-B d M$.

3. Preliminary results. The main result of this section, Theorem 1 , will be used in $\$ 4$ to construct homeomorphisms from $E^{3}$ onto $E^{3}$ having certain properties. Lemmas 1 and 2 are preliminary results for Theorem 1 .

Suppose that $C$ is a polyhedral 3-cell in $E^{3}$. If $x$ and $y$ are distinct points of Bd $C$, the statement that $\alpha$ is an unknotted chord of $C$ from $x$ to $y$ means that $\alpha$ is a polygonal arc with endpoints $x$ and $y$ such that (1) (Int $\alpha) \subset \operatorname{Int} C$ and (2) if $\beta$ is any polygonal arc on $\operatorname{Bd} C$ from $x$ to $y$, then $\alpha \cup \beta$ is the boundary of a polyhedral disc $D$ such that (Int $D) \subset$ Int $C$. It may be shown [20] that if $\alpha$ and $\alpha^{\prime}$ are two unknotted chords of the polyhedral 3-cell $C$ such that $\alpha$ and $\alpha^{\prime}$ have the same endpoints, there is a homeomorphism $h$ from $C$ onto $C$ such that if $p \in \operatorname{Bd} C, h(p)=p$ and $h[\alpha]=\alpha^{\prime}$.

LEMMA 1. If $C$ is a polyhedral 3-cell in $E^{3}, x$ and $y$ are distinct points of $\mathrm{Bd} C$, and $B$ is a compact 0 -dimensional subset of $C$ containing neither $x$ nor $y$, then there is an unknotted chord $\alpha$ of $C$ such that $\alpha$ has endpoints $x$ and $y$ and is disjoint from $B$.

Proof. We may assume that $C$ is the cube

$$
\left\{(x, y, z): \quad(x, y, z) \in E^{3}, \quad|x| \leqq 1,|y| \leqq 1, \text { and }|z| \leqq 1\right\},
$$


that $B$ intersects neither the top nor bottom face of $C$, and that $x$ and $y$ belong to the top and bottom faces, respectively, of $C$. Let $D$ be the common part of $C$ and the $X Z$-plane. $D$ is a disc in $C$ such that $B d D \subset \operatorname{Bd} C$, and $x$ and $y$ lie on Bd $D$.

Since $B$ is a compact 0 -dimensional set, $B \cap D$ does not separate $x$ and $y$ in $D$. Hence there is polygonal arc $\alpha$ on $D$ such that $\alpha$ has endpoints $x$ and $y$, $\alpha$ is disjoint from $B$, and (Int $\alpha) \subset$ Int $D$. It is easy to see that $\alpha$ is an unknotted chord of $C$.

The proof of Lemma 1 given above was shown to the author by R. H. Bing and is simpler than the author's original argument.

The following lemma is proved in [19].

Lemma 2. If $G$ is a point-like decomposition of $E^{3}$ such that $E^{3} / G$ is homeomorphic to $E^{3}$ and $U$ is a simply connected open set in $E^{3} / G$, then $P^{-1}[U]$ is simply connected.

We may now establish the main result of this section.

THEOREM 1. Suppose that $G$ is a point-like decomposition $E^{3}$ such that $E^{3} / G$ is homeomorphic to $E^{3}$ and $\mathrm{Cl} P\left[H_{G}\right]$ is a compact 0-dimensional set. Suppose that $M$ is a compact polyhedral 3-manifold-with-boundary in $E^{3}$ such that $\mathrm{Bd} M$ is connected and is disjoint from $\mathrm{Cl} H_{G}$. Then there is a homeomorphism $h$ from $M$ onto $P[M]$ such that $h|\mathrm{Bd} M=P| \mathrm{Bd} M$.

Proof. Let $K$ denote $P[\operatorname{Bd} M] ; K$ is homeomorphic to $B d M$. Since $E^{3} / G$ is homeomorphic to $E^{3}, K$ has exactly one bounded complementary domain $U$ in $E^{3} / G$. Let $N$ denote $K \cup U$. It may be shown that $P[M]=N$.

Since Bd $M$ has a cartesian product neighborhood in $E^{3}, K$ has a cartesian product neighborhood in $E^{3} / G$. Hence $N$ is a compact 3-manifold-with-boundary, $\operatorname{Bd} N=P[\operatorname{Bd} M]$, and Int $N=P[\operatorname{Int} M]$.

Since Theorem 1 is easily proved if $\mathrm{Bd} M$ is a 2 -sphere, we assume that $\mathrm{Bd} M$ is not a 2-sphere. Let $T_{0}$ be a triangulation of $N[11],[16]$ such that if $\sigma$ is any 3-simplex of $T_{0}$ and $S_{2}$ is the carrier of the 2-skeleton on $T_{0}$, then $S_{2}-\mathrm{Bd} \sigma$ is connected. Let $v_{1}, v_{2}, \cdots$, and $v_{k}$ be the vertices of $T_{0}$ lying in Int $N$. Let $B_{1}, B_{2}, \cdots$, and $B_{k}$ be mutually disjoint open balls such that if $i=1,2, \cdots$, or $k, v_{i} \in B_{i}$ and $B_{i} \subset$ Int $N$. If $i=1,2, \cdots$, or $k$, then, since $\mathrm{Cl} P\left[H_{G}\right]$ is 0-dimensional, $B_{i}-\mathrm{Cl} P\left[H_{G}\right]$ exists, and let $v_{i}^{*}$ be a point of $B_{i}-\mathrm{Cl} P\left[H_{G}\right]$. There is a homeomorphism $f_{0}$ from $N$ onto $N$ such that if $x \in N$ and $x \notin \bigcup_{i=1}^{k} B_{i}$, then $f_{0}(x)=x$, and if $i=1,2, \cdots$, or $k, f_{0}\left[B_{i}\right]=B_{i}$ and $f_{0}\left(v_{i}\right)=v_{i}^{*}$. Let $T_{1}$ be $\left\{f_{0}[\sigma]: \sigma \in T_{0}\right\}$. Then $T_{1}$ is a triangulation of $N$, no vertex of $T_{1}$ belongs to $\mathrm{Cl} P\left[H_{G}\right]$, and if $\sigma$ is any 3-simplex of $T_{1}$ and $S_{2}^{\prime}$ is the carrier of the 2-skeleton of $T_{1}$, then $S_{2}^{\prime}-\mathrm{Bd} \sigma$ is connected.

If no 1-simplex of $T_{1}$ intersects $\mathrm{Cl} P\left[H_{G}\right]$, let $T$ be $T_{1}$. Otherwise, let $s_{1}, s_{2}, \cdots$, and $s_{m}$ be the 1-simplexes of $T_{1}$ which intersect $\mathrm{Cl} P\left[H_{G}\right]$. There exist mutually 
disjoint polyhedral(relative to $T_{1}$ ) 3-cells $R_{1}, R_{2}, \cdots$, and $R_{m}$ such that if $i=1,2, \cdots$, or $m$, then (1) $\left(s_{i} \cap \mathrm{Cl} P\left[H_{G}\right]\right) \subset \operatorname{Int} R_{i}$, (2) $R_{i} \subset \operatorname{Int} N$, and (3) $s_{i} \cap R_{i}$ is an unknotted chord of $R_{i}$. Such 3-cells may be constructed in the following way: If $i=1,2, \cdots$, or $m$, let $s_{i}^{\prime}$ be a subarc of $s_{i}$, lying in Int $s_{i}$, and such that $\left(s_{i}^{\prime} \cap \mathrm{Cl} P\left[H_{G}\right]\right) \subset \operatorname{Int} s_{i}^{\prime}$. Since $s_{i}^{\prime}$ is polygonal (relative to $T_{1}$ ), it may be thickened slightly to give $R_{i}$.

If $i=1,2, \cdots$, or $m$, let $x_{i}$ and $y_{i}$ be the two points of $s_{i} \cap \operatorname{Bd} R_{i}$. By Lemma 1 , there is an unknotted chord $t_{i}$ of $R_{i}$ from $x_{i}$ to $y_{i}$ missing $\mathrm{Cl} P\left[H_{G}\right]$. There is, then, a homeomorphism $k_{i}$ from $R_{i}$ onto $R_{i}$ such that if $p \in \operatorname{Bd} R_{i}, k_{i}(p)=p$ and $k_{i}\left[s_{i} \cap R_{i}\right]=t_{i}$. It is clear that there is a homeomorphism $k$ from $N$ onto $N$ such that (1) if $x \notin \bigcup_{i=1}^{m} R_{i}, k(x)=x$ and (2) if $i=1,2, \cdots$, or $m$, and $x \in R_{i}, k(x)=k_{i}(x)$. Let $T$ be $\left\{k[\sigma]: \sigma \in T_{1}\right\}$. Then $T$ is a triangulation of $N$ such that the carrier $\Sigma_{1}$ of the 1-skeletion of $T$ is disjoint from $\mathrm{Cl} P\left[H_{G}\right]$. Let $\Sigma_{2}$ denote the carrier of the 2-skeleton of $T$.

Let $\Delta_{1}, \Delta_{2}, \cdots$, and $\Delta_{r}$ be the 2-simplexes of $T$ whose interiors lie in Int $N$. If $i=1,2, \cdots$, or $r$, there is an annulus $A_{i}$ on $\Delta_{i}$ such that (1) $A_{i}$ is disjoint from $\mathrm{Cl} P\left[H_{G}\right]$ and (2) $\mathrm{Bd} \Delta_{i}$ is one boundary component of $A_{i}$. If $i=1,2, \cdots$, or $r$, let $J_{i}$ be a centerline of $A_{i}$ and let $D_{i}$ be the subdisc of $\Delta_{i}$ having $J_{i}$ as its boundary; $D_{i} \cap A_{i}$ is an annulus $B_{i}$. Let $V_{1}, V_{2}, \cdots$, and $V_{r}$ be mutually disjoint simply connected open sets such that if $i=1,2, \cdots$, or $r$, then (1) $\mathrm{Cl} V_{i} \subset$ Int $N$, (2) $V_{i} \cap \Sigma_{2}=$ Int $D_{i}$, and (3) $\mathrm{Cl} V_{i} \cap \Sigma_{2}=D_{i}$. Such open sets may be constructed by slight thickenings of Int $D_{1}$, Int $D_{2}, \cdots$, and Int $D_{r}$.

If $i=1,2, \cdots$, or $r$, let $U_{i}$ be $P^{-1}\left[V_{i}\right]$. By Lemma 2, each of $U_{1}, U_{2}, \cdots$, and $U_{r}$ is simply connected. It is easily seen, with the aid of Dehn's lemma [18] that if $i=1,2, \cdots$, or $r$, there is a polyhedral disc $E_{i}$ such that (1) Int $E_{i} \subset U_{i}$ and (2) $\operatorname{Bd} E_{i}=P^{-1}\left[J_{i}\right]$. Note that $E_{i} \subset M$. If $i=1,2, \cdots$, or $r$, let $Y_{1}$ be $E_{i} \cup P^{-1}\left[A_{i}-B_{i}\right] ; Y_{i}$ is a disc with boundary $P^{-1}\left[\Delta_{i}\right]$, and $Y_{i} \subset M$.

If $i$ and $j$ are distinct positive integers, neither greater than $r$, then Int $Y_{i}$ and Int $Y_{j}$ are disjoint, for (1) Int $A_{i}$ and Int $A_{j}$ are disjoint and (2) $U_{i}$ and $U_{j}$ are disjoint.

If $i=1,2, \cdots$, or $r$, let $L_{i}$ denote the closure of $\left(A_{i}-B_{i}\right)$. It is clear that $P^{-1} \mid\left[(\operatorname{Bd} N) \cup\left(\bigcup_{i=1}^{r} L_{i}\right)\right]$ is a homeomorphism $h_{0}$ from $(\operatorname{Bd} N) \cup\left(\bigcup_{i=1}^{r} L_{i}\right)$ onto $(\mathrm{Bd} M) \cup\left(\bigcup_{i=1}^{r} P^{-1}\left[L_{i}\right]\right)$ such that $h_{0}\left|\mathrm{Bd} N=P^{-1}\right| \mathrm{Bd} N$. It is easily seen that there is a homeomorphism $g$ from $\Sigma_{2}$ onto $(\mathrm{Bd} M) \cup\left(\bigcup_{i=1}^{r} Y_{i}\right)$ such that (1) $g\left|\operatorname{Bd} N=P^{-1}\right| \operatorname{Bd} N$ and (2) if $i=1,2, \cdots$, or $r, g\left[\Delta_{i}\right]=Y_{i}$. It is clear that $g\left[\Sigma_{2}\right] \subset M$.

Suppose that $\sigma$ is a 3-simplex of $T$ and let $S$ be $\mathrm{Bd} \sigma$. We shall show now that $g\left[\Sigma_{2}\right]$ is disjoint from Int $g[S]$, the interior, in $E^{3}$, of the 2-sphere $g[S]$.

Suppose that there is a point $p$ of $\Sigma_{2}$ such that $g(p) \in \operatorname{Int} g[S]$. Clearly $p \in \Sigma_{2}-S$. Now $T$ has the property that if $\sigma$ is any 3 -simplex of $T$, then $\Sigma_{2}-\operatorname{Bd} \sigma$ is connected. Therefore $\Sigma_{2}-S$ is connected. Consequently $g\left[\Sigma_{2}\right] \subset(g[S] \cup \operatorname{Int} g[S])$, and thus $\mathrm{Bd} M \subset(g[S] \cup \operatorname{Int} g[S])$. Since $\mathrm{Bd} M$ is not a 2-sphere, there is a point 
of $g[S]$ not in $M$. This is a contradiction, for $g\left[\Sigma_{2}\right] \subset M$. Hence, no point of $g\left[\Sigma_{2}\right]$ lies in Int $g[S]$.

Let $K_{1}, K_{2}, \cdots$, and $K_{n}$ be the distinct 2-spheres in $N$ which are boundaries of 3 -simplexes of $T$. Let $W$ be $\left\{q\left[K_{i}\right] \cup \operatorname{Int} g\left[K_{i}\right]: i=1,2, \cdots\right.$, or $\left.n\right\}$. We shall show now that $\mathrm{W}$ is a triangulation of $M$.

First, $W$ is a 3-complex. For suppose that $i$ and $j$ are distinct positive integers, neither greater than $n$. Then Int $g\left[K_{i}\right]$ and Int $g\left[K_{j}\right]$ are disjoint. For, it was shown that $g\left[K_{i}\right]$ and Int $g\left[K_{j}\right]$ and Int $g\left[K_{j}\right]$ are disjoint, and so are $g\left[K_{j}\right]$ and Int $g\left[K_{i}\right]$. If Int $g\left[K_{j}\right]$ and Int $g\left[K_{j}\right]$ intersect, then one contains the other, say Int $g\left[K_{i}\right] \subset \operatorname{Int} g\left[K_{j}\right]$. Since $g\left[K_{i}\right] \neq g\left[K_{j}\right]$, then $g\left[K_{i}\right]$ intersects Int $g\left[K_{j}\right]$. This is a contradiction, and hence Int $g\left[K_{i}\right]$ and Int $g\left[K_{j}\right]$ are disjoint. Since $g\left[K_{i}\right] \cap g\left[K_{j}\right]=g\left[K_{i} \cap K_{j}\right]$, it is clear that $W$ is a 3-complex.

Second, $\bigcup\{w: w \in W\}=M$. Since $g\left[\Sigma_{2}\right] \subset M$, it is clear that

$$
\bigcup\{w: w \in W\} \subset M .
$$

Hence we need only to show that $M \subset \bigcup\{w: w \in W\}$.

Suppose that $M=\bigcup\{w: w \in W)$ exists. Since $\bigcup\{w: w \in W\}$ is closed, there is a point $p$ of Int $M$ such that $p \notin \bigcup\{w: w \in W\}$. Let $q$ be a point such that for some 3-simplex $w$ of $W, q \in \operatorname{Int} w$. Then $q \in \operatorname{Int} M$ and there is an $\operatorname{arc} p q$ in Int $M$; we may assume that $p q$ is disjoint from the carrier of the 1-skeleton of $W$. Let $b$ be the first point of $\bigcup\{w: w \in W\}$ on $p q$ in the order from $p$ to $q ; p b-\{b\}$ is disjoint from $\bigcup\{w: w \in W\}$. There is a 2 -simplex $\tau$ of $W$ such that $b \in \operatorname{Int} \tau$, and there is a 3-simplex $\sigma$ of $W$ such that $\tau \subset \operatorname{Bd} \sigma$. Now $\tau \notin \operatorname{Bd} M$. For if $\tau \subset \operatorname{Bd} M$, then clearly the $\operatorname{arc} p b$ intersects $E^{3}-M$, which is impossible. Thus $\tau \notin \operatorname{Bd} M$. It follows that $g^{-1}[\tau] \notin \mathrm{Bd} N$.

There is exactly one 3-simplex $t_{1}$ of $T$ such that $\mathrm{Bd} \sigma=g\left[\mathrm{Bd} t_{1}\right]$. There is a 3-simplex $t_{2}$ of $T$ such that Int $t_{1}$ and Int $t_{2}$ are disjoint and $\mathrm{Bd} t_{1} \cap \mathrm{Bd} t_{2}=g^{-1}[\tau]$. Let $\sigma^{\prime}$ be $g\left[\mathrm{Bd} t_{2}\right] \cup \operatorname{Int} g\left[\mathrm{Bd} t_{2}\right]$. Then $\sigma^{\prime} \in W$ and $\sigma \cap \sigma^{\prime}=\tau$. Since Int $\sigma$ and Int $\sigma^{\prime}$ are disjoint and $\mathrm{Bd} \sigma \cap \operatorname{Bd} \sigma^{\prime}=\tau$, it follows that $(p b-\{b\}) \subset \operatorname{Int} \sigma^{\prime}$. This is a contradiction, and it follows that $M=\bigcup\{w: w \in W\}$.

Consequently, $W$ is a triangulation of $M$. It is clear that there is an extension $f$ of $g$ such that (1) $f$ is a homeomorphism from $N$ onto $M$ and (2) $f \mid \operatorname{Bd} N=$ $P^{-1} \mid \operatorname{Bd} N$. It follows that there is a homeomorphism $h$ from $M$ onto $N$ such that $h|\operatorname{Bd} M=P| \operatorname{Bd} M$. This concludes the proof of Theorem 1 .

4. Results on point-like decompositions. In this section, we establish the main results of the paper relative to point-like decompositions $G$ of $E^{3}$ such that $E^{3} / G$ either is homeomorphic to $E^{3}$ or is a 3-manifold.

THEOREM 2. Suppose that $G$ is a point-like decomposition of $E^{3}$ such that (1) $E^{3} / G$ is homeomorphic to $E^{3}$ and (2) $\mathrm{Cl} P\left[H_{G}\right]$ is a compact 0 -dimensional set. Suppose that $U$ is an open set in $E^{3}$ containing $\mathrm{Cl}_{G}$ and $\varepsilon$ is a positive 
number. Then there is a homeomorphism $h$ from $E^{3}$ onto $E^{3}$ such that (1) if $x \notin U, h(x)=x$ and (2) if $g$ is any nondegenerate element of $G,(\operatorname{diam} h[g])<\varepsilon$.

Proof. Since $\mathrm{Cl} P\left[H_{G}\right]$ is a compact 0-dimensional subset of $E^{3} / G$ and $E^{3} / G$ is homeomorphic to $E^{3}$, there exists a sequence $N_{1}, N_{2}, N_{3}, \cdots$ such that (1) for each positive integer $i, N_{i}$ is a compact polyhedral 3-manifold-with-boundary such that (a) each component of $N_{i}$ has connected boundary and diameter less than $1 / i$, and (b) $N_{i+1} \subset \operatorname{Int} N_{i}$, and (2) $\mathrm{Cl} P\left[H_{G}\right]=\bigcup_{i=1}^{\infty} N_{i}$. For each positive integer $i$, let $M_{i}$ denote $P^{-1}\left[N_{i}\right] ; M_{i}$ is a compact 3-manifold-with-boundary. Since, for each positive integer $i, N_{i}$ is polyhedral, then $M_{i}$ is tame in $E^{3}$.

Since $\mathrm{Cl}_{G} \subset U, P[U]$ is open in $E^{3} / G$ and there is, accordingly, a positive integer $k$ such that $N_{k} \subset P[U]$. It follows that $M_{k} \subset U$. Let $M_{k 1}, M_{k 2}, \cdots$, and $M_{k m}$ be the components of $M_{k}$. If $j=1,2, \cdots$, or $m$, there is, by Theorem 1, a homeomorphism $f_{j}$ from $M_{k j}$ onto $P\left[M_{k j}\right]$ such that $f_{j}\left|\operatorname{Bd} M_{k j}=P\right| \operatorname{Bd} M_{k j}$. Let $f$ be the homeomorphism from $M_{k}$ onto $P\left[M_{k}\right]$ such that if $j=1,2, \cdots$, or $m, f \mid M_{k j}=f_{j}$. Now $f^{-1}$ is uniformly continuous and there exists a positive integer $r$ greater than $k$ such that if $L$ is any component of $P\left[M_{r}\right]$, then (diam $\left.f^{-1}[L]\right)<\varepsilon$. Let $M_{r 1}, M_{r 2}, \cdots$, and $M_{r n}$ be the components of $M_{r}$. With the aid of Theorem 1, it follows that if $i=1,2, \cdots$, or $n$, there is a homeomorphism $k_{i}$ from $M_{r i}$ onto $f^{-1}\left[P\left[M_{r i}\right]\right]$ such that $k_{i}\left|\operatorname{Bd} M_{r i}=f^{-1} P\right| \operatorname{Bd} M_{r i}$. Let $k$ be the homeomorphism from $M_{r}$ onto $f^{-1}\left[P\left[M_{r}\right]\right]$ such that if $i=1,2, \cdots$, or $n, k \mid M_{r i}=k_{i}$.

Define a homeomorphism $h$ as follows: (1) If $x \notin M_{k}$, then $h(x)=x$. (2) If $x \in\left(M_{k}-M_{r}\right)$, then $h(x)=f^{-1}(P(x))$. (3) If $x \in M_{r}$, then $h(x)=k(x)$. Then $h$ is a homeomorphism from $E^{3}$ onto $E^{3}$. If $x \notin U$, then since $x \notin M_{k}, h(x)=x$. If $g$ is any nondegenarate element of $G$, then for some positive! integer $i$ not greater than $n, g \subset M_{r i}$. Hence $h[g] \subset h\left[M_{r i}\right]$; but $h\left[M_{r i}\right]=f^{-1}\left[P\left[M_{r i}\right]\right]$, and $\left.\left(\operatorname{diam} f^{-1}\left[P M_{r i}\right]\right]\right)<\varepsilon$. Therefore $(\operatorname{diam} h[g])<\varepsilon$ and the proof of Theorem 2 is complete.

The following theorem may be compared with Theorem 2 of [1].

THEOREM 3. Suppose that $G$ is a point-like decomposition of $E^{3}$ such that $P\left[H_{G}\right]$ is a compact 0 -dimensional set. Then $E^{3} / G$ is homeomorphic to $E^{3}$ if and only if for each open set $U$ in $E^{3}$ containing $H_{G}$ and each positive number $\varepsilon$, there is a homeomorphism $h$ from $E^{3}$ onto $E^{3}$ such that (1) if $x \notin U, h(x)=x$ and (2) if $g$ is any nondegenerate element of $G,(\operatorname{diam} h[g])<\varepsilon$.

Proof. If, for each open set $U$ in $E^{3}$ containing $H_{G}$ and each positive number $\varepsilon$, there exists a homeomorphism $h$ from $E^{3}$ onto $E^{3}$ having the properties stated above, then it follows from the proof of Theorem 1 of [4] that $E^{3} / G$ is homeomorphic to $E^{3}$. Hence the condition stated is sufficient. By Theorem 2, it is necessary.

The following theorem may be compared with Theorem 5 of [1]. 
ThEOREM 4. Suppose that $G$ is a point-like decomposition of $E^{3}$ such that $P\left[H_{G}\right]$ is a compact 0 -dimensional set. If $E^{3} / G$ is a 3-manifold, then $E^{3} / G$ is homeomorphic to $E^{3}$.

Proof. If $E^{\mathbf{3}} / G$ is a 3-manifold, then a local version of Theorem 1 holds. Hence the proof of Theorem 2 is valid and thus the conclusion of that theorem holds. Then by Theorem $3, E^{3} / G$ is homeomorphic to $E^{3}$.

Corollary 1. If $G$ is a point-like decomposition of $S^{3}$ such that $P\left[H_{G}\right]$ is a compact 0-dimensional set and $S^{3} / G$ is a 3-manifold, then $S^{3} / G$ is a 3-sphere.

5. Monotone decompositions of $E^{3}$. In this section and the next, we study monotone decompositions $G$ of $E^{3}$ such that (1) $P\left[H_{G}\right]$ is contained in a compact 0-dimensional set and (2) $E^{3} / G$ is homeomorphic to $E^{3}$. We want to determine conditions under which it can be concluded that each element of $G$ is point-like.

A compact continuum $K$ in $E^{3}$ is cellular if and only if there exists a sequence $C_{1}, C_{2}, C_{3}, \cdots$ of 3 -cells in $E^{3}$ such that (1) if $n$ is any positive integer, $C_{n+1} \subset$ Int $C_{n}$, and (2) $K=\bigcap_{i=1}^{\infty} C_{i}$. It is well known that for compact continua in $E^{3}$, "point-like", and "cellular"' are equivalent; see [21].

THEOREM 5. Suppose that $G$ is a monotone decomposition of $E^{3}$ such that $P\left[H_{G}\right]$ is contained in a compact 0 -dimensional set and $E^{3} / G$ is homeomorphic to $E^{3}$. Suppose that if $U$ is any open set in $E^{3}$ containing $\mathrm{Cl} H_{G}$ and $\varepsilon$ is any positive number, there exists a homeomorphism from $E^{3}$ onto $E^{3}$ such that (1) if $x \notin U, f(x)=x$, and (2) if $g$ is any nondegenerate element of $G$, then $(\operatorname{diam} f[g])<\varepsilon$. Then each element of $G$ is point-like.

Proof. Since $\mathrm{Cl} P\left[H_{G}\right]$ is compact and 0-dimensional, there exists a sequence $N_{1}, N_{2}, N_{3}, \cdots$ of compact 3-manifolds-with-boundary in $E^{3} / G$ such that (1) if $i$ is any positive integer, $N_{i+1} \subset$ Int $N_{i}$, and each component of $N_{i}$ has diameter less than $1 / i$, and (2) $\bigcap_{i=1}^{\infty}=P\left[H_{G}\right]$. For each positive integer $i$, let $M_{i}$ be $P^{-1}\left[N_{i}\right]$. Then for each positive integer $i, M_{i}$ is a compact 3-manifold-withboundary and $M_{i+1} \subset$ Int $M_{i}$. Further, $\bigcap_{i=1}^{\infty} M_{i}=\mathrm{Cl} H_{G}$.

Suppose that $g$ is a nondegenerate element of $G$. For each positive integer $i$, let $K_{i}$ be the component of $M_{i}$ containing $g$. Then for each positive integer $i$, $K_{i}$ is a compact 3-manifold-with-boundary, and $K_{i+1} \subset \operatorname{Int} K_{i}$. Further $\bigcap_{i=1}^{\infty} K_{i}=g$.

Suppose now that $j$ is a positive integer. We shall show that there is a 3-cell $C$ such that $g \subset$ Int $C$ and $C \subset$ Int $K_{j}$. Let $\left\{R_{1}, R_{2}, \cdots, R_{m}\right\}$ be a finite set of 3-cells, each contained in Int $K_{j}$ and such that $\left\{\right.$ Int $R_{1}$, Int $R_{2}, \cdots$, Int $\left.R_{m}\right\}$ covers $K_{j+1}$. There exists a positive number $\varepsilon$ such that if $A$ is any subset of $K_{j+1}$ of diameter less than $\varepsilon$, then $A$ is contained in some one of Int $R_{1}$, Int $R_{2}, \cdots$, and Int $R_{m}$. By hypothesis, there is a homeomorphism $f$ from $E^{3}$ onto $E^{3}$ such that (1) if $x \notin$ Int $M_{j+1}$, then $f(x)=x$ and $(2)(\operatorname{diam} f[g])<\varepsilon$. It follows that $f\left[K_{j+1}\right] \subset K_{j+1}$, and hence there is a positive integer $k$ not greater than $m$ such that $f[g] \subset \operatorname{Int} \mathbf{R}_{k}$. Let $C$ be $f^{-1}\left[R_{k}\right]$; clearly $C$ is a 3 -cell such that $g \subset \operatorname{Int} C$ and $C \subset \operatorname{Int} K_{j}$. 
It now follows that $g$ is cellular. There exists a 3-cell $C_{1}$ such that $g \subset$ Int $C_{1}$ and $C_{1} \subset$ Int $K_{1}$. Let $n_{1}$ be 1 . There is a positive integer $n_{2}$ such that $K_{n_{2}} \subset \operatorname{Int} C_{1}$. There is a 3-cell $C_{2}$ such that $g \subset \operatorname{Int} C_{2}$ and $C_{2} \subset \operatorname{Int} K_{n_{2}}$. A continuation of this process yields an increasing sequence $n_{1}, n_{2}, n_{3}, \cdots$ of positive integers and a sequence $C_{1}, C_{2}, C_{3}, \cdots$ of 3 -cells such that for each positive integer $i$, $g \subset$ Int $C_{i}$ and $C_{i} \subset$ Int $K_{n_{i}}$. It is clear that for each positive integer $i, C_{i+1} \subset \operatorname{Int} C_{i}$, and that $\bigcap_{i=1}^{\infty} C_{i}=g$. Hence $g$ is cellular. Hence $g$ is point-like and Theorem 5 is established.

The following theorem is closely related to Theorem 1 . In the proof of Theorem 6 we construct triangulations in the order opposite to that used in the proof of Theorem 1.

THEOREM 6. Suppose that $G$ is a monotone decomposition of $E^{3}$ such that (1) $E^{3} / G$ is homeomorphic to $E^{3}$ and (2) $\mathrm{Cl} P\left[H_{G}\right]$ is a compact 0 -dimensional set. Suppose that $M$ is a compact polyhedral 3-manifold-with-boundary in $E^{3}$ such that $\mathrm{Bd} M$ is a connected and is disjoint from $H_{G}$. Suppose that $M$ has a triangulation $T$ such that the carrier $\Sigma_{1}$ of the 1-skeleton of $T$ is disjoint from $\mathrm{Cl} H_{G}$. Then there is a homeomorphism h from $M$ onto $P[M]$ such that $h|\mathrm{Bd} M=P| \mathrm{Bd} M$.

Proof. Let $\Delta_{1}, \Delta_{2}, \cdots$, and $\Delta_{n}$ be the 2-simplexes of $T$ that intersect Int $M$. If $i=1,2, \cdots$, or $n$, let $F_{i}$ be a disc in Int $\Delta_{i}$ and such that Int $F_{i}$ contains $\Delta_{i} \cap \mathrm{Cl}_{G}$. If $i=1,2, \cdots$, or $n$, there is an annulus $A_{i}$ on $\Delta_{i}$ such that

$$
\mathrm{Bd} A_{i}=\left(\mathrm{Bd} \Delta_{i}\right) \cup\left(\mathrm{Bd} F_{i}\right) ;
$$

let $\gamma_{i}$ be a centerline of $A_{i}$ and let $D_{i}$ be the subdisc of $\Delta_{i}$ bounded by $\gamma_{i}$. Note that $D_{i} \cap A_{i}$ is an annulus $B_{i}$, and that $A_{i}$ is disjoint from $\mathrm{Cl} H_{G}$.

Let $N$ be $P[M]$; it may be shown that $N$ is a compact 3-manifold-with-boundary, Bd $N=P[\operatorname{Bd} M]$, and Int $N=P[$ Int $M]$. If $i=1,2, \cdots$, or $n$, then $P\left[D_{i}\right]$ is a singular disc lying in Int $N$, and having no singularities on $P\left[B_{i}\right]$. Further, there is a neighborhood $U$ of $\Sigma_{1}$ such that (1)P $\mid\left[U \cup\left(\bigcup_{i=1}^{n} A_{i}\right)\right]$ is a homeomorphism and (2) if $x \notin U \cup\left(\bigcup_{i=1}^{n} A_{i}\right.$, then $P(x) \notin P\left[U \cup\left(\bigcup_{i=1} A_{i}\right)\right]$.

With the aid of Dehn's lemma [18], it may be shown that there exists discs $K_{1}, K_{2}, \cdots$, and $K_{n}$ such that (1) if $i=1,2, \cdots$, or $n$, (a) $K_{i} \subset \operatorname{Int} N$, (b) $\operatorname{Bd} K_{i}$ $=P\left[\operatorname{Bd} D_{i}\right]$, and (c) $K_{i} \cap P\left[U \cup\left(\bigcup_{j=1}^{n} A_{j}\right)\right] \subset P\left[B_{i}\right]$, and (2) if $! i$ and $j$ are distinct positive integers, neither greater than $n$, then $K_{i}$ and $\mathrm{Bd} K_{j}$ are disjoint.

The discs $K_{1}, K_{2}, \cdots$, and $K_{n}$ are not necessarily mutually disjoint. By an argument similar to that given in the proof of Theorem 9 of [7], it may be shown that there exist mutually disjoint discs $K_{1}^{\prime}, K_{2}^{\prime}, \cdots$, and $K_{n}^{\prime}$ such that if $i=1,2, \cdots$, or $n, \mathrm{Bd} K_{i}^{\prime}=\mathrm{Bd} K_{i}, K_{i}^{\prime} \subset$ Int $N$, and $K_{i}^{\prime}$ is disjoint from $P\left[\bigcup_{j=1}^{n}\left(A_{j}-B_{j}\right)\right]$. Then if $i=1,2, \cdots$, or $n, K_{i}^{\prime} \cup P\left[A_{i}-B_{i}\right]$ is a disc $\Delta_{i}^{\prime}$.

By an argument similar to that used in the proof of Theorem 1, it may be 
shown that there is a homeomorphism $h$ from $M$ onto $P[M]$ such that $h|\operatorname{Bd} M=P| \operatorname{Bd} M$. This completes the proof of Theorem 6 .

Corollary 2. Suppose that $G$ is a monotone decomposition of $E^{3}$ such that (1) $E^{3} / G$ is homeomorphic to $E^{3}$ and (2) $\mathrm{Cl} P\left[H_{G}\right]$ is a compact 0-dimensional set. Suppose that there exists a sequence $M_{1}, M_{2}, M_{3}, \cdots$ of compact 3-manifolds-with-boundary in $E^{3}$ such that (1) if $n$ is any positive integer, $M_{n} \subset M_{n+1}$ and $M_{n}$ has a triangulation such that the carrier of its 1-skeleton is disjoint from $\mathrm{Cl} H_{G}$, and (2) $\bigcap_{i=1}^{\infty} M_{i}=\mathrm{Cl}_{G}$. Then each element of $G$ is point-like.

Proof. By an argument similar to that given to prove Theorem 2, but using Theorem 6 in place of Theorem 1, we may show that under the hypothesis of the corollary, the following holds: If $U$ is any open set in $E^{3}$ containing $H_{G}$ and $\varepsilon$ is any positive number, there exists a homeomorphism $h$ from $E^{3}$ onto $E^{3}$ such that (1) if $x \notin U, h(x)=x$, and (2) if $g$ is any nondegenerate element of $G$, then (diam $h[g]$ ) $<\varepsilon$. It then follows by Theorem 5 that each element of $G$ is point-like.

6. Decompositions of $E^{3}$ into continua of type $T$. The statement that a compact metric continuum $M$ is of type $T$ means that if $K$ is any subcontinuum of $M$ that can be embedded in a 2-sphere and $f$ is an embedding of $K$ in some 2-sphere $S$, then $f[K]$ does not separate $S$. Since for planar continua, separating the plane is a topological invariant, the criterion used is meaningful. Continua of type $T$ include tree-like continua [8], snake-like continua [8], dendrons, and arcs.

THEOREM 7. If $G$ is a monotone decomposition of $E^{3}$ such that (1) $E^{3} / G$ is homeomorphic to $E^{3}$, (2) $P\left[H_{G}\right]$ is contained in a compact 0-dimensional set, and (3) each element of $G$ is a continuum of type $T$, then each element of $G$ is point-like.

Proof. First it will be shown that if $M$ is a compact polyhedral 3-manifoldwith-boundary in $E^{3}$ such that $\mathrm{Bd} M$ is connected and is disjoint from $\mathrm{Cl} H_{G}$, then $M$ has a triangulation $L$ such that the carrier of the 1-skeleton of $L$ is disjoint from $\mathrm{Cl} H_{G}$.

Let $L_{0}$ be any triangulation of $M$. Let $v_{1}, v_{2}, \cdots$, and $v_{n}$ denote the vertices of $L_{0}$. Since $\mathrm{Cl} P\left[H_{G}\right]$ is compact and 0 -dimensional, there are points $v_{1}^{\prime}, v_{2}^{\prime}, \cdots$,

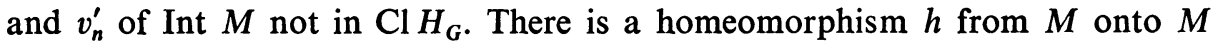
such that $h \mid \mathrm{Bd} M$ is the identity and if $i=1,2, \cdots$, or $n, h\left(v_{i}\right)=v_{i}^{\prime}$. Let $L_{1}$ denote $\left\{h[\sigma]: \sigma \in L_{0}\right\} . L_{1}$ is a triangulation of $M$ and no vertex of $L_{1}$ belongs to $\mathrm{Cl} \mathrm{H}_{\mathrm{G}}$.

If $s$ is a 1-simplex of $L_{1}$, let $C_{s}$ be a 3-cell containing $s \cap \mathrm{Cl} H_{G}$, obtained by a slight thickening of a subarc $s^{\prime}$ of $s$ and such that $C_{s} \cap s=s^{\prime}$. It is to be true that if $s$ and $t$ are distinct 1-simplexes of $L_{1}, C_{s}$ and $C_{t}$ are disjoint. Now by hypothesis, if $g_{0}$ is any subcontinuum of an element of $G, g_{0}$ does not separate any 
2-sphere containing $g_{0}$. In addition, each component of $\left(\mathrm{Bd} C_{s}\right) \cap \mathrm{Cl} H_{G}$ is a subcontinuum of some element of $G$. Hence no component of $\left(\mathrm{Bd} C_{s}\right) \cap \mathrm{Cl} H_{G}$ separates $\mathrm{Bd} C_{s}$. By unicoherence, $\left(\mathrm{Bd} C_{s}\right)-\left(\mathrm{Cl} C_{s}\right)$ is connected. There is, therefore, an arc $s^{\prime \prime}$ on $\mathrm{Bd} C_{s}$, disjoint from $\mathrm{Cl} H_{G}$ and having as endpoints the endpoints of $s^{\prime}$. It is now easy to construct a triangulation $L$ of $M$ such that

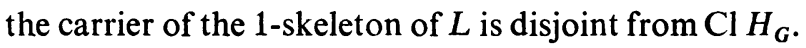

By using Theorem 6 in place of Theorem 1, the argument used to establish Theorem 2 shows that the hypothesis of Theorem 5 is satisfied. Hence by Theorem 5 , each element of $G$ is point-like.

COROLlaRY 3. If $G$ is a monotone decomposition of $E^{3}$ such that (1) $E^{3} / G$ is homeomorphic to $E^{3}$, (2) $P\left[H_{G}\right]$ is contained in a compact 0-dimensional set, and (3) each element of $G$ is a tree-like continuum, then each element of $G$ is point-like.

COROLlaRY 4. If $G$ is a monotone decomposition of $E^{3}$ into arcs and one-point sets such that $E^{3} / G$ is homeomorphic to $E^{3}$ and $\mathrm{Cl} P\left[H_{G}\right]$ is a compact 0 -dimensional set, then each element of $G$ is point-like $\left({ }^{2}\right)$.

7. Results on point-like decompositions. In this section, we present some results on point-like decompositions of $E^{3}$. We begin with two results on 3-cells-withhandles.

The statement that $K$ is a 3-cell-with-handles means that $K$ is an orientable 3-manifold-with-boundary such that there exist 3-cells $C_{0}, C_{2}, \cdots$, and $C_{n}$ such that (1) any two of $C_{1}, C_{2} \cdots$ and $C_{n}$ are disjoint, (2) if $i=1,2, \cdots$, or $n$, $C_{0} \cap C_{i}=\left(\mathrm{Bd} C_{0}\right) \cap\left(\mathrm{Bd} C_{i}\right)$ and $C_{0} \cap C_{i}$ is the union of two disjoint discs, and (3) $K=\bigcup_{i=0}^{n} C_{i}$.

Lemma 3. If $K$ is a polyhedral 3-cell-with-handles and $L$ is a polyhedral 3-cell such that $K \cap L=(\operatorname{Bd} K) \cap(\operatorname{Bd} L)$ and $K \cap L=D_{1} \cup D_{2} \cup \cdots \cup D_{n}$ where $D_{1}, D_{2}, \cdots$, and $D_{n}$ are mutually disjoint polyhedral discs, then $K \cup L$ is a polyhedral 3-cell-with-handles.

LEMMA 4. If $B$ is a compact 0-dimensional subset of $E^{3}$ and $U$ is any open set containing $B$, there exists a polyhedral 3-manifold-with-boundary $M$ such that $B \subset$ Int $M, M \subset U$, and each component of $M$ is a 3-cell-with-handles.

Proof. There exists a polyhedral 3-manifold-with-boundary $N$ such that $B \subset N$ and $N \subset U$. It follows with the aid of Lemma 1 that there is a triangulation $T$ of $N$ such that the carrier of the 1-skeleton of $T$ is disjoint from $B$.

Let $N_{0}$ be a component of $N$, and let $S_{0}$ be the carrier of the 1-skeleton of the triangulation $T_{0}$ of $N_{0}$ induced by $T$. There exists a polyhedral tubular neighborhood $S_{0}^{*}$ of $S_{0}$ such that (1) $S_{0}^{*}$ is disjoint from $B$, (2) if $\sigma$ and $\sigma^{\prime}$ are distinct

(2) Joseph M. Martin has recently established this result without requiring that $\mathrm{Cl} P\left[H_{G}\right]$ be compact and 0-dimensional. 
3-simplexes of $T_{0}$ and $\sigma-\left(\operatorname{Int} S_{0}^{*}\right)$ and $\sigma^{\prime}-\left(\right.$ Int $\left.S_{0}^{*}\right)$ intersect, then their common part is a disc lying in the interior of some 2-simplex of $T_{0}$, and (3) $N_{0}-\operatorname{Int} S_{0}^{*}$ is connected. With the aid of Lemma 3, it follows that $N_{0}-\operatorname{Int} S_{0}^{*}$ is a 3-cell-withhandles. Lemma 4 now follows easily.

Suppose that $G$ is an upper semicontinuous decomposition of $E^{3}$. The statement that $H_{G}$ is definable by 3-cell-with-handles means that there exists a sequence $M_{1}, M_{2}, M_{3}, \cdots$ such that (1) for each positive integer $n, M_{n}$ is a polyhedral 3-manifold-with-boundary such that each component of $M_{n}$ is a 3-cell-with-handles and $M_{n+1} \subset$ Int $M_{n}$, (2) $\mathrm{Cl} H_{G}=\bigcap_{i=1}^{\infty} M_{i}$, and (3) $g$ is a nondegenerate element of $G$ if and only if $g$ is a nondegenerate component of $\bigcap_{i=1}^{\infty} M_{i}$. It is clear that if $H_{G}$ is definable by 3-cells-with-handles, then $\mathrm{Cl} P\left[H_{G}\right]$ is a compact 0-dimensional set.

THEOREM 8. Suppose that $G$ is a point-like decomposition of $E^{3}$ such that (1) $E^{3} / G$ is homeomorphic to $E^{3}$ and (2) $\mathrm{Cl} P\left[H_{G}\right]$ is a compact 0-dimensional set. Then $H_{G}$ is definable by 3-cells-with-handles.

Proof. Since $E^{3} / G$ is homeomorphic to $E^{3}$, there is, by Lemma 4 , a sequence $M_{1}, M_{2}, M_{3}, \cdots$ such that (1) for each positive integer $i, M_{i}$ is a compact polyhedral 3-manifold-with-boundary, $M_{i+1} \subset \operatorname{Int} M_{i}$, and each component of $M_{i}$ is a 3-cell-with-handles, and (2) $\mathrm{Cl} P\left[H_{G}\right]=\bigcap_{i=1}^{\infty} M_{i}$. It is clear that $\mathrm{Cl} H_{G}=\bigcap_{i=1}^{\infty} P^{-1}\left[M_{i}\right]$.

Suppose that $j$ is a positive integer and $L$ is a component of $M_{j}$. Since $L$ is a 3-cell-with-handles, there exist polyhedral 3-cells $C_{0}, C_{1}, \cdots$, and $C_{r}$ such that (1) any two of $C_{1}, C_{2}, \cdots$, and $C_{r}$ are disjoint, (2) if $i=1,2, \cdots$, or $r$,

$$
C_{0} \cap C_{i}=\left(\operatorname{Bd} C_{0}\right) \cap\left(\operatorname{Bd} C_{i}\right)
$$

and $C_{0} \cap C_{1}$ is the union of two disjoint polyhedral discs $D_{i}^{\prime}$ and $D_{i}$, and (3) $L=\bigcup_{i=0}^{r} C_{i}$. An argument similar to that given in proving Theorem 1 may be used to show that there exists 3-cells $C_{0}^{\prime}, C_{1}^{\prime}, \cdots$, and $C_{r}^{\prime}$ such that $\bigcup_{i=0}^{r} C_{i}^{\prime}=P^{-1}[L]$ and $C_{0}^{\prime}, C_{1}^{\prime}, \cdots$, and $C_{r}^{\prime}$ satisfy conditions relative to $P^{-1}[L]$ analogous to those satisfied by $C_{0}, C_{1}, \cdots$, and $C_{r}$ relative to $L$. It follows that $P^{-1}[L]$ is a 3-cellwith-handles. It is easily seen that $H_{G}$ is definable by 3-cells-with-handles.

The following result, which was announced in [3], provides a converse to Theorem 8. It may be proved by line of argument similar to that used to establish Theorem 6 and Corollary 2.

THEOREM 9. Suppose that $G$ is a monotone decomposition of $E^{3}$ such that (1) $E^{3} / G$ is homeomorphic to $E^{3}$ and (2) $P\left[H_{G}\right]$ is a compact 0 -dimensional set. If $H_{G}$ is definable by 3-cells-with-handles, then each element of $G$ is point-like.

Our last result concerns the construction of a point-like decomposition of $E^{3}$ for which the associated decomposition space has certain given properties. 
THEOREM 10. If $K$ is any compact 0-dimensional subset of $E^{3}$, there exist a point-like decomposition $G$ of $E^{3}$ and a homeomorphism $h$ from $E^{3} / G$ onto $E^{3}$ such that $h\left[P\left[H_{G}\right]\right]=K$.

Proof. Let $M_{1}, M_{2}, M_{3}, \cdots$ be a sequence of polyhedral 3-manifolds-withboundary such that (1) if $i$ is any positive integer, $M_{i+1} \subset$ Int $M_{i}$, and (2) $\bigcap_{i=1}^{\infty} M_{i}=K$. Let $P_{1}$ and $P_{2}$ be disjoint planes in $E^{3}$.

There is a homeomorphism $h_{1}$ from $E^{3}$ onto $E^{3}$ such that if $M_{1}^{\prime}$ is any component of $M_{1}, h_{1}\left[\right.$ Int $\left.M_{1}^{\prime}\right]$ intersects both $P_{1}$ and $P_{2}$. There is a homeomorphism $h_{2}$ from $E^{3}$ onto $E^{3}$ such that if $x \varepsilon E^{3}-$ Int $M_{1}, h_{2}(x)=x$ and if $M_{2}^{\prime}$ is any component of $M_{2}$, then $h_{2} h_{1}\left[\right.$ Int $\left.M_{2}^{\prime}\right]$ intersects both $P_{1}$ and $P_{2}$. Suppose that $i$ is a positive integer and $h_{1}, h_{2}, \cdots$, and $h_{i}$ have been defined. There is a homeomorphism $h_{i+1}$ from $E^{3}$ onto $E^{3}$ such that if $x \in E^{3}-$ Int $M_{i}, h_{i+1}(x)=x$, and if $M_{i+1}^{\prime}$ is any component of $M_{i+1}$, then $h_{i+1} h_{i} \cdots h_{1}$ [Int $\left.M_{i+1}^{\prime}\right]$ intersects both $P_{1}$ and $P_{2}$. Therefore there exists a sequence $h_{1}, h_{2}, h_{3}, \cdots$ of homeomorphisms from $E^{3}$ onto $E^{3}$ having properties indicated above. For each positive integer $i$, let $N_{i}$ be $h_{i} h_{i-1} \cdots h_{1}\left[M_{i}\right]$.

Let $G$ be the decomposition of $E^{3}$ whose nondegenerate elements are the components of $\bigcap_{i=1}^{\infty} N_{i}$. It may be proved, using [17, Chapter V, Theorem 20], that $G$ is upper semicontinuous. Note that $H_{G}=\bigcap_{i=1}^{\infty} N_{i}$.

Now we shall show that if $U$ is any open set in $E^{3}$ containing $H_{G}$ and $\varepsilon$ is any positive number, there is a homeomorphism $f$ from $E^{3}$ onto $E^{3}$ such that if $x \notin U, f(x)=x$ and if $g$ is any nondegenerate element of $G$, (diam $f[g])<\varepsilon$. Suppose $U$ is open in $E^{3}$ and $\varepsilon$ is any positive number. There is a positive integer $j$ such that $N_{j} \subset U$. Since (1) for each positive integer $n, M_{n}$ is compact, (2) $h_{j} h_{j-1} \cdots h_{1}$ is a homeomorphism, and (3) the maximum of the diameters of the components of $M_{n}$ approaches 0 as $n$ increases without bound, there is a positive integer $k$ greater than $j$ such that if $M_{k}^{\prime}$ is any component of $M_{k}^{\prime}$, $\left(\right.$ diam $\left.h_{j} h_{j-1} \cdots h_{1}\left[M_{k}^{\prime}\right]\right)<\varepsilon$. Let $f$ be $\left(h_{k} h_{k-1} \cdots h_{j+1}\right)^{-1}$. Then $f\left[N_{k}\right]=h_{j} h_{j-1} \cdots h_{1}\left[M_{k}\right]$ and if $x \in E^{3}-N_{j}$, then $f(x)=x$. Hence $f$ is a homeomorphism from $E^{3}$ onto $E^{3}$ having the specified properties.

It may now be seen, from a consideration of the proof of Theorem 1 of [4], that there is a homeomorphism $h$ from $E^{3} / G$ onto $E^{3}$ such that $h\left[P\left[H_{G}\right]\right]=K$. With the aid of Theorem 5, it may be proved that each element of $G$ is point-like.

\section{REFERENCES}

1. S. Armentrout, Upper semi-continuous decompositions of $E^{3}$ with at most countably many non-degenerate elements, Ann. of Math. 78 (1963), 605-618.

2. Concerning point-like decompositions of $S^{3}$ that yield 3-manifolds, Abstract 619-115, Notices Amer. Math. Soc. 12 (1965), 90.

3. R. J. Bean, Decompositions of $E^{3}$ which yield E3, Abstract 619-198, Notices Amer. Math. Soc. 12 (1965), 117.

4. R. H. Bing, Upper semicontinuous decompositions of E3, Ann. of Math. 65 (1957), 363-374. 
5. - A decomposition of $E^{3}$ into points and tame arcs such that the decomposition space is topologically different from E3, Ann. of Math. 65 (1957), 484-500.

6. $-A$ homeomorphism between the 3-sphere and the sum of two solid horned spheres, Ann. of Math. 59 (1952), 354-362.

7. - Point like decompositions of $E^{2}$, Fund. Math. 50 (1962), 431-453.

8. —_, Snake-like continua, Duke Math. J. 18 (1951), 653-663.

9. - Inequivalent families of periodic homeomorphisms of E3, Ann. of Math. 80 (1964), 78-93.

10. - Topology of 3-manifolds and related topics, Decompositions of $E^{3}$, PrenticeHall, Englewood Cliffs, N. J., 1962; pp. 5-21.

11. —, An alternative proof that 3-manifolds can be triangulated, Ann. of Math. 69 (1959), 37-65.

12. K. W. Kwun, Upper semi-continuous decompositions of the n-sphere, Proc. Amer. Math. Soc. 13 (1962), 284-290.

13. K. W. Kwun and F. Raymond, Almost acyclic maps of manifolds, Amer. J. Math. 86 (1964), 638-650.

14. L. L. Lininger, The sum of two crumpledcubes is $S^{3}$ if it is a 3-manifold, Abstract 64T-445, Notices Amer. Math. Soc. 11 (1964), 678.

15. L. F. McAuley, Another decomposition of $E^{3}$ into points and intervals (to appear).

16. E. E. Moise, Affine structures in 3-manifolds. V. The triangulation theorem and Hauptvermutung, Ann. of Math. 56 (1952), 92-114.

17. R. L. Moore, Foundations of point set theory, rev. ed., Amer. Math. Soc. Colloq. Publ. Vol. 13, Amer. Math. Soc., Providence, R. I., 1962,

18. C. D. Papakyriakopoulos, On Dehn's lemma and the asphericity of knots, Ann. of Math. 66 (1957), 1-250.

19. T. M. Price, Cellular decompositions of E3, Ph. D. Thesis, University of Wisconsin, Madison, Wis., 1964.

20. H. Schubert, Knoten und Vollringe, Acta Math. 90 (1953), 132-286.

21. D. G. Stewart, Cellular subsets of the 3-sphere, Trans. Amer. Math. Soc. 114 (1965), $10-22$.

UNIVERSITY OF IOWA,

Iowa Cirt, IOWA

INSTITUTE FOR ADVANCED STUDY,

Princeton, New Jersey 